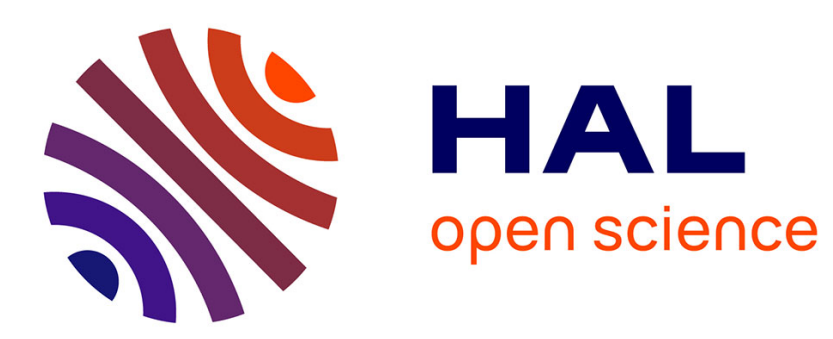

\title{
La liaison de prédications en ancien français: le cas des subordonnées circonstancielles en tête d'énoncé
}

Bernard Combettes

\section{To cite this version:}

Bernard Combettes. La liaison de prédications en ancien français: le cas des subordonnées circonstancielles en tête d'énoncé. Langue française, 2014, 182, pp.123-136. hal-01102704

\author{
HAL Id: hal-01102704 \\ https://hal.science/hal-01102704
}

Submitted on 8 Jan 2016

HAL is a multi-disciplinary open access archive for the deposit and dissemination of scientific research documents, whether they are published or not. The documents may come from teaching and research institutions in France or abroad, or from public or private research centers.
L'archive ouverte pluridisciplinaire HAL, est destinée au dépôt et à la diffusion de documents scientifiques de niveau recherche, publiés ou non, émanant des établissements d'enseignement et de recherche français ou étrangers, des laboratoires publics ou privés. 


\title{
La liaison de prédications en ancien français : le cas des subordonnées circonstancielles en tête d'énoncé
}

\author{
Linking between predications in Old French: \\ the case of fronted adverbial clauses
}

\author{
Bernard COMBETTES \\ Université de Lorraine \& UMR-ATILF
}

\begin{abstract}
Résumé :
Le but de cette étude est de déterminer le statut syntaxique de certaines propositions circonstancielles placées en tête d'énoncé en ancien français. Il s'agit en particulier d'évaluer leur position sur le continuum allant de la parataxe à l'hypotaxe. On examine deux types de faits qui peuvent servir d'indices de plus ou moins grande intégration syntaxique : l'expression du sujet et sa position dans le contexte de droite, le jeu des formes verbales, avec l'alternance du présent et du passé simple dans des propositions successives. On observe en conclusion que les propositions temporelles de simultanéité ou de postériorité sont moins intégrées syntaxiquement que les autres circonstancielles (causales, finales, comparatives et temporelles d'antériorité).
\end{abstract}

Mots-clefs : proposition circonstancielle, proposition temporelle, concordance des temps, place du sujet, parataxe, hypotaxe

\begin{abstract}
:
The purpose of this study is to determine the syntactic status of fronted adverbial clauses in Old French narrative texts, observing their position on the continuum from parataxis to hypotaxis. We examine two types of facts which indicate syntactic integration degree of the clause: the expression of the subject and its position in the main clause, the temporal sequence, with alternation of present and simple past in successive clauses. We come to the conclusion that temporal clauses expressing simultaneity or posteriority are syntactically less embedded than other adverbial clauses (causal, final, comparative and temporal expressing anteriority).
\end{abstract}

Keywords: adverbial clause, temporal clause, sequence of tenses, subject position, parataxis, hypotaxis

Dans les études sur la liaison de prédications et sur le statut des propositions qu'elle concerne, la construction d'un continuum de subordination est une tendance bien représentée, en particulier dans certaines approches fonctionnelles. Cette conception de « degrés de subordination » est en grande partie justifiée par le fait que sont d'ordinaire pris en considération plusieurs paramètres qui ne vont pas obligatoirement dans le même sens. Si de telles tentatives - on pense par exemple aux propositions de Lehmann (1988) ou au travail de Van Valin \& LaPolla (1997) - s'en tiennent d'ordinaire à un point de vue synchronique, il semble toutefois possible d'interpréter ces échelles en diachronie, l'orientation du continuum étant alors à considérer comme la représentation de la direction du changement et les degrés de l'échelle de subordination comme des étapes que l'évolution doit parcourir. Sans discuter ici sur le fond l'approche par continuum (cf. Debaisieux 2013 : 43-47), c'est dans ce cadre que nous voudrions développer notre étude, la notion de degrés d'intégration et les propriétés prises en compte nous semblant conduire à des observations intéressantes sur des états de langue anciens. Problématique double, en réalité : nous tenterons d'une part de déterminer dans quelle mesure la diachronie du français fournit des matériaux permettant d'alimenter l'argumentation sur l'existence d'un continuum de subordination, de degrés de syntactisation ; nous nous attacherons d'autre part, dans une perspective plus fonctionnelle, à dégager les aspects discursifs qu'il convient de mettre en relation avec ce type de liaison de prédications.

\section{Liaison de prédications et continuum}


Avant d'examiner les données de l'ancien français, nous présenterons d'abord rapidement deux types d'analyse en continuum. Le premier, développé par Lehmann, s'appuie sur des critères d'ordre morphosyntaxique, alors que le deuxième, composante du modèle élaboré par Van Valin \& LaPolla, repose essentiellement sur l'interface du domaine syntaxique et de la sémantique des événements.

Désirant mettre en lumière des corrélations entre divers paramètres afin d'établir une typologie de la liaison (linkage) des propositions, Lehmann (1988) prend en compte six variables, chacune d'elles se réalisant sous la forme d'un continuum :

- degré d'intégration de la subordonnée

- point d'incidence de la subordonnée (portée sur le verbe, sur le SV, sur la phrase)

- perte des caractéristiques phrastiques

- grammaticalisation du verbe principal

- degré d'« entrelacement » (interlacing) entre les deux propositions

- explicitation de la dépendance (présence d'un morphème subordonnant)

Dans notre perspective, les points qui nous intéressent plus particulièrement ici sont le degré d'intégration et le degré d'entrelacement, les autres caractéristiques apparaissant davantage comme des sortes de conséquences de ces deux critères principaux. La détermination de l'intégration d'une subordonnée dans la phrase matrice accorde une importance particulière, parmi d'autres critères, à la plus ou moins grande liberté de position des propositions et on considère en effet habituellement qu'un faible degré d'intégration va de pair avec des contraintes moins fortes dans la linéarisation.

C'est la combinaison de ces diverses échelles qui permet de situer la liaison de prédications par rapport aux deux extrêmes constitués par la parataxe et par l'hypotaxe la plus nette, correspondant à l'enchâssement. Ce continuum se trouve ainsi construit, de façon prioritaire, sur des critères morphosyntaxiques, les aspects sémantiques et discursifs n'étant pris en compte qu'indirectement et de façon très marginale.

La position adoptée par Van Valin \& LaPolla (1997), dans une problématique explicitement fonctionnelle, est évidemment différente, puisqu'il s'agit de mettre en relation le continuum des degrés de liaison et des caractéristiques relevant du contenu. Le degré de liaison sémantique dépend de la façon dont une construction donnée exprime les divers aspects, les «facettes », d'un événement ou, au contraire, renvoie à des événements distincts, à des états de chose indépendants (Van Valin \& LaPolla 1997 : 478). Ce degré est supposé aller de pair avec le degré de liaison syntaxique : dans le cas de la parataxe, par exemple, chaque proposition sera caractérisée par une forte autonomie sémantique, alors que l'hypotaxe correspondra à une relation plus étroite. On peut voir là la mise en œuvre du principe d'iconicité, l'interdépendance - plus ou moins forte - des états de chose auxquels renvoient les prédications se reflétant dans la structuration syntaxique de l'énoncé. L'application de ce critère conduit à l'élaboration d'une échelle dont l'une des extrémités, correspondant aux cas les moins intégrés, les plus proches de la parataxe, comprend trois degrés : l'hypothèse, la temporalité, les états de chose non ordonnés ; le domaine de la temporalité est lui-même organisé en degrés correspondant aux diverses relations chronologiques :

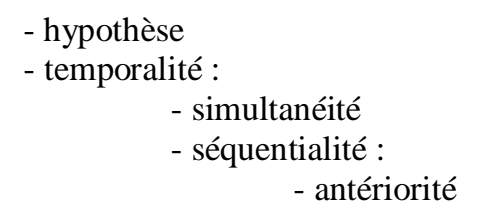




\section{- succession immédiate (du type dès que...) \\ - succession avec intervalle (un moment après que...) \\ - états de chose non ordonnés}

(Van Valin \& LaPolla, 1997, 476 sq.)

Du point de vue syntaxique, les propositions qui occupent la zone extrême du continuum ont un très faible degré d'intégration, ce qui irait dans le même sens que le critère de l'entrelacement de Lehmann. Si l'on s'en tient à la famille traditionnelle des propositions « circonstancielles », on constatera que les temporelles et les hypothétiques se distingueraient ainsi des finales et des causales, davantage intégrées à l'ensemble de l'énoncé complexe.

D'une manière générale, on peut noter que les aspects discursifs sont très peu pris en compte, mais, dans la suite de notre étude, nous tenterons de montrer que, dans le cas des propositions les moins intégrées du moins, la dimension textuelle est un paramètre aussi important que les caractéristiques strictement sémantiques et qu'il convient de la faire intervenir en tant que telle dans la détermination des degrés de la dépendance syntaxique.

Dans la partie qui va suivre, nous essayerons de déterminer quels sont les indices qui vont dans le sens des hiérarchisations que nous venons de rappeler, pour voir si certains aspects spécifiques de l'ancien français permettent d'illustrer un tel continuum de subordination ou s'il convient d'y apporter des modifications. Dans la mesure où une rupture assez nette s'établit entre les propositions régies, qui présentent les degrés les plus élevés d'intégration et de dépendance, et les propositions qui ont le statut de constituants périphériques, d'ordinaire considérées comme des «circonstancielles », nous nous attacherons essentiellement à ces dernières. L'une des principales raisons de ce choix réside dans le fait que la relation avec le domaine discursif relève, pour ces propositions non dépendantes, d'une problématique particulière, alors que les propositions dépendantes ne peuvent être traitées de la même manière, sur ce point du moins, cette différence étant en partie à mettre en rapport avec la plus ou moins grande liberté de position de la proposition dans la structure globale de l'énoncé.

Le corpus sur lequel est basé cette étude est constitué de deux textes narratifs du XIII ${ }^{\mathrm{e}}$ siècle, l'un, La Conqueste de Constantinople, de J. de Villehardouin, qui relève du genre de la chronique, dans lequel nous prendrons la plupart de nos exemples, l'autre qui fait partie d'un cycle de romans en prose, Les Sept Sages de Rome. Ce choix a été en grande partie guidé par le fait que nous disposons pour ces deux textes d'éditions qui restituent fidèlement la ponctuation du manuscrit ${ }^{1}$. Même s'il est difficile, lorsqu'il s'agit d'ancien français, de s'appuyer sur des indices fournis par la segmentation graphique, dans la mesure où celle-ci, si elle n'est pas absente, ne paraît pas constituer un système assez cohérent pour que l'on puisse l'exploiter de façon satisfaisante, nous verrons qu'il est parfois possible d'observer certaines tendances qui peuvent soutenir l'interprétation des faits linguistiques.

\section{Les faits de langue}

Nous allons examiner à présent la situation telle qu'elle se présente en ancien français en privilégiant les faits de langue relevant de la linéarisation des constituants, faits qui paraissent le mieux traduire le degré de liaison des propositions. Notons tout d'abord que ces faits qui nous semblent pertinents sont à considérer comme des conséquences du degré - plus ou moins élevé - de dépendance. Il ne s'agit donc pas, à proprement parler, de propriétés définitoires telles que celles que Lehmann propose de distinguer, mais de caractéristiques plus

\footnotetext{
${ }^{1}$ Josfroy de Vileharduyn, La conqueste de Costentinoble, Editions du CRAL, Université de Nancy 2, 1978. Les Sept Sages de Rome, Edition du CRAL, Université de Nancy 2, 1981.
} 
secondaires, si l'on peut dire, dont la combinaison peut mettre en lumière le statut des relations syntaxiques.

\subsection{L'ordre des constituants}

Ce critère a un statut particulier, dans la mesure où c'est la structure générale de l'énoncé qui se trouve concernée, les autres propriétés que nous examinerons par la suite n'étant pas d'ordre syntaxique, mais relevant du domaine discursif ou du champ énonciatif. Les caractéristiques qui apparaissent comme dignes d'intérêt sont liées à la disposition des constituants dans la zone initiale du contexte de droite qui suit la proposition initiale. Le raisonnement d'ordinaire adopté, relativement simple, consiste à considérer que, dans un système syntaxique qui est, sous beaucoup d'aspects, proche du type V2 (cf. Skarup 1975, Marchello-Nizia 1995), si une séquence XVY ne donne aucune indication sur la fonction de $X$, qui peut être un circonstanciel ou un élément régi, une séquence XYVZ, en revanche, permet de conclure au statut périphérique de $\mathrm{X}$, qui se trouve en quelque sorte en dehors de l'unité prédicative soumise à la règle du verbe second. Cette idée générale peut être appliquée au cas particulier des propositions « subordonnées » placées en tête d'énoncé.

\subsubsection{Les propositions hypothétiques}

Reprenant la zone inférieure du continuum que nous avons présenté plus haut, nous nous arrêterons d'abord sur le cas des propositions hypothétiques, qui sont considérées comme plus dépendantes que les temporelles, dans la mesure où une corrélation, ne serait-ce qu'au plan sémantique, semble s'établir entre les deux prédications. Dans un texte comme la Conqueste de Constantinople, la position d'une telle subordonnée ne paraît pas apporter de modifications au contexte de droite. La deuxième proposition, qui constitue l'apodose, correspond au type V2 ; elle peut présenter la séquence $\mathrm{SV}$, avec sujet nominal ou pronominal :

(1) Car se ceste ost remaint la rescousse d'outremer est faillie (Villehardouin, p. 13)

Car si cette armée tarde, l'aide aux terres d'outremer est perdue

(2) Se vous le mandiez que il venist [...] je croi que il le feroit (p. 9)

Si vous lui demandiez qu'il vienne, je crois qu'il le ferait

ou, plus rarement, une séquence XVS, comme dans l'exemple suivant, où l'ordre participe + auxiliaire s'accompagne de la postposition du syntagme sujet :

(3) Car se Diex n'i eust mis conseil destruite fust la crestienté (p. 66)

Car si Dieu n'y eût mis bonne entente, la chrétienté ê̂t été détruite

Si l'on excepte un exemple de non-expression du sujet dans un tour impersonnel :

(4) Et se tu y entres ne me samble mie que tu le face pour mon preu (p. 63)

Et si tu y entres, il ne me semble pas que tu le fasses à mon avantage

on ne relève pas d'occurrences d'une séquence se $\mathrm{P}, \mathrm{VX}$, dans laquelle le verbe principal suivrait immédiatement la subordonnée, ce qui serait l'indice d'une intégration plus forte de la proposition initiale. Cette constatation va à l'encontre de l'analyse adoptée dans les travaux que nous avons résumés plus haut, qui placent les hypothétiques à un degré intermédiaire entre les cas de dépendance forte et les cas plus proches de la parataxe. Ce statut particulier peut être considéré comme le résultat de la spécificité que l'on pourrait qualifier de «polyphonique» qui caractérise les hypothétiques, la relation qui unit les propositions s'accompagnant, de façon plus ou moins nette, d'une relation entre les actes de parole. Un exemple comme :

(5) Se nous alons en Surie l'entree de l'iver est quant nous y vendrons et ne porrons ostoier (p. 44)

Si nous allons en Syrie, le début de l'hiver est quand nous y viendrons et nous ne pourrons guerroyer 
fait bien apparaître la portée sur l'énonciation, le lien de causalité qu'implique l'hypothèse pouvant être paraphrasé par : Si nous allons en Syrie, (on doit rappeler que) l'entrée [...]

Les hypothétiques se trouvent ainsi dans une situation intermédiaire : le mouvement énonciatif tend à donner une certaine autonomie aux propositions, alors que la corrélation sémantique maintient un lien relativement fort entre les deux composantes de l'énoncé. Ce statut se reflète dans la ponctuation, la liaison sémantique semblant l'emporter, puisque les hypothétiques initiales ne sont séparées du contexte de droite que dans $17 \%$ des cas. Le choix de la segmentation ne paraît pas motivé et on remarquera par exemple que, contrairement à ce que l'on pourrait attendre, la longueur de la subordonnée n'entraîne pas obligatoirement l'emploi du point :

(6) Se vous le mandiez que il venist ca et preist la croiz et se meist ou leu le conte de Champaigne et li donissiez la seignorie de l'ost je croi que il le feroit (p. 9)

Se vous lui demandiez qu'il vienne ici et qu'il prenne la croix et qu'il prenne la place du Comte de Champagne et si vous lui donniez le commandement de l'armée, je crois qu'il le ferait

\subsubsection{Les propositions temporelles}

En ce qui concerne les propositions temporelles, on notera d'abord le comportement particulier des subordonnées en quant, de loin les plus représentées dans la prose narrative. Le schéma type, qui peut être considéré comme une routine discursive (cf. Rychner 1970), est constitué de la séquence quant $\mathrm{P}$, si $\mathrm{VX}$, le sujet n'étant d'ordinaire pas exprimé dans la mesure où il renvoie à un référent thématique qui n'a pas à être réactivé. L'introducteur de proposition si peut alterner avec d'autres adverbiaux comme lors :

(7) Quant il oirent que li marchis venoit si alerent encontre lui (p. 9)

Quand ils apprirent que le marquis venait, ils allèrent à sa rencontre

(8) Quant cil de Rome les en virent foir lors corurent après (Sept Sages, p. 53)

Quand ceux de Rome les virent s'enfuir, ils leur coururent après

S’il est exprimé, le syntagme sujet peut se trouver postposé :

(9) Et quant la feste fu passee si parla li emperieres de ses aferes (Villehardouin, p. 60)

Et quand la fête fut passée, l'empereur parla de ses affaires

Il peut également précéder le verbe dans un schéma quant $\mathrm{P}$, Sujet + Verbe :

(10) Quant il les vouldrent asaillir cil dedenz quirent plet coment il se rendroient (p. 89)

Quand ils voulurent les assaillir, ceux de dedans prirent conseil pour savoir comment ils se rendraient

Ce qu'il faut souligner, c'est que toutes ces variantes vont dans le même sens, dans la mesure où, comme dans le cas des hypothétiques, n'est pas utilisé le schéma quant $\mathrm{P}, \mathrm{V}(\mathrm{S})$, dans lequel la proposition initiale serait à proximité immédiate du verbe et entraînerait éventuellement la postposition du sujet. En ce sens, la subordonnée en quant apparaît comme moins intégrée dans la structure propositionnelle que les constituants nominaux. On peut par exemple comparer les deux énoncés suivants :

(11) L'andemain ou au tierz jour manda li dus [...] XL des plus sages homes (p. 6)

Le lendemain, au troisième jour, le duc convoqua les quarante hommes ...

(12) Quant la messe fu dite li dus manda aus messages que [...] (p. 6)

Quand la messe fut dite, le duc ordonna aux messagers que [...]

La situation n'est sans doute pas symétrique, certains circonstants nominaux n'entrainant pas l'inversion, comme dans l'exemple suivant, où la présence d'une structure propositionnelle dans le syntagme initial peut justifier l'ordre SV du contexte de droite :

(13) Au terme que li dus leur mist il vindrent au palés (p. 5) 
À la date que le duc leur fixa, ils vinrent au palais

Il n'en reste pas moins que les propositions en quant font preuve d'une nette homogénéité. L'ajout d'autres constituants à proximité de la subordonnée ne modifie pas la structuration de l'ensemble de l'énoncé. Ainsi, l'extraction du sujet en position de topique, comme dans :

(14) Et Henri quant il sot sa venue si prist conseil et dit [...] (p. 74)

Et Henri, quand il apprit sa venue, tint conseil et dit [...]

(15) et ces povres fames qui ces petiz enfanz avoient quant eles ne pooient entrer chiés ces riches homes qui $[. .$.$] à cel feu se chaufoient [...] (Sept Sages, p. 35)$

et ces pauvres femmes qui avaient ces petits enfants, quand elles ne pouvaient pas entrer chez ces riches qui $[. .$.$] , se chauffaient à ce feu [\ldots]$

ne change-t-elle pas l'organisation en V2 du prédicat principal.

Comparé à cette régularité, le statut des autres temporelles est moins nettement tranché. Les locutions dont le sémantisme correspond, avec diverses nuances, à celui de la simultanéité ou de la postériorité présentent un fonctionnement proche de celui de quant $\mathrm{P}$, mais de façon moins homogène. Considérons par exemple le cas des propositions introduites par endementres que, qui expriment la simultanéité ; dans la plupart des exemples sont appliquées les mêmes règles que pour les propositions en quant :

(16) Endementieres que il ala parler aus contes et aus barons. Icele partie dont [...] parlerent aus messages (p. 18)

Pendant qu'il alla parler aux comtes et aux barons, cette partie dont [...] parla aux messagers

(17) Endementieres que li emperieres fu en cele ost si vint une moult grande mesaventure (p. 45)

Pendant que l'empereur fut dans ce camp, arriva une grande mésaventure

mais l'énoncé suivant, malgré, si l'on peut dire, la segmentation par le point, présente l'ordre VS dans le contexte de droite :

(18) Endementieres que li mesages vindrent. estoient $\mathrm{V}$ nés chargiees au port de Costentinoble de pelerins et de chevaliers $[\ldots]$ (p. 85)

Pendant que les messages vinrent, cinq nefs étaient remplies de pèlerins et de chevaliers

Dans les textes que nous avons observés, ce sont les propositions temporelles renvoyant à l'antériorité qui présentent un fonctionnement particulier. Alors que les subordonnées en quant s'insèrent dans un contexte de type V2, la position des subordonnées d'antériorité placées en tête d'énoncé va de pair avec le schéma conj. $+\mathrm{P}+\mathrm{VX}$, dans lequel elles occupent la zone initiale et se comportent comme un constituant entraînant l'ordre V (S) X. Dans le texte de Villehardouin, la locution einz que (avant que) entraîne systématiquement cette linéarisation :

(19) ainz qu'il peussent estre armé en ocirent il assez (p. 76) avant qu'ils puissent s'armer, ils en tuèrent un grand nombre

(20) Mes eincois que l'estour parfenist vint I chevalier [...] (p. 36)

Mais avant que le combat se termine vint un chevalier

Si l'on suit le raisonnement que nous avons adopté, on considérera ces subordonnées comme des propositions dotées d'un degré d'intégration plus élevé que les autres temporelles. Il est intéressant de les comparer, sur ce point, aux causales ou aux comparatives par exemple. On peut en effet constater que les propositions introduites par por ce que ou par einsi (autresi) comme entrent également dans une structuration identique de l'énoncé :

(21) Et pour ce que il sevent que [...] vous prient por dieu que [...] (p. 5)

Et parce qu'ils savent que [...] ils vous prient que [...]

(22) Autresi conme cel palés fu renduz au marchis [...] Fu renduz cil de Blasquerne a Henri (Villehardouin, p. 57)

De la même manière que ce palais fut rendu au marquis [...] celui de Blaquerne fut rendu à Henri 
Il convient d'observer ici, en prenant en compte les aspects morphosyntaxiques, la formation des locutions conjonctives. Alors que la forme quand, héritée du latin quando, n'implique pas de corrélation particulière avec une autre forme, des locutions comme tant que dans le domaine temporel, aussi / si comme pour la comparaison ou encore par ce que pour la causalité, sont le résultat de la grammaticalisation de tours corrélatifs mettant en jeu des adverbes ou des syntagmes prépositionnels, qui, dans la structure de départ, entretiennent une relation de dépendance avec la prédication principale. On rappellera les cas de tmèse, comme dans les deux exemples suivants (cités par Buridant $2000: 610,624$ ), où ainz et por ce sont disjoints de la conjonction que :

(23) Mais ainz fu la novele dite / Au chastel que il venissent (Yvain)

Mais la nouvelle fut dite au château avant qu'ils arrivent

(24) Car por ce vos a Diex envoié entre nos, que vos parfaçoiz ce que [...] (Queste du Graal)

Car Dieu vous a envoyé parmi nous pour que vous accomplissiez ce que [...]

Ce type de linéarisation ne peut que renforcer la tendance à faire de la que $\mathrm{P}$ un constituant placé dans la zone postverbale de l'énoncé, comme le développement du circonstant représenté par l'adverbe ou par le syntagme prépositionnel. Le degré d'intégration de ces subordonnées, plus dépendantes que les temporelles en quant, se trouve ainsi en accord, si l'on peut dire, avec le type de subordonnant.

Les propositions marquant l'antériorité forment ainsi une sous-catégorie particulière à l'intérieur des temporelles ; plus proches des finales ou des causales, elles sont caractérisées par un degré d'intégration syntaxique plus élevé que les propositions de simultanéité ou de postériorité. Cette différence ne semble pas être prise en considération dans les échelles de subordination, ce qui n'a rien de surprenant, dans la mesure où l'examen des propriétés et des critères considérés comme pertinents s'effectue dans le cadre phrastique. La prise en compte des relations sémantiques (dépendance entre les états de chose dénotés, entrelacement) conduirait même à des résultats opposés aux observations que nous pouvons faire ici. Considérant que, de façon symétrique, la relation d'antériorité et celle de postériorité dissocient plus fortement les événements dénotés que la relation de simultanéité, Van Valin \& LaPolla donnent en effet aux propositions de «succession » un degré d'intégration plus faible qu'aux propositions de concomitance. Nous verrons plus loin (§3) que l'examen du fonctionnement de ces propositions dans un contexte large, en prenant en considération leur rôle discursif, permet de justifier les différences que nous venons de constater.

\subsection{Le jeu des temps verbaux}

Le fonctionnement particulier des propositions temporelles, dont nous venons d'examiner, avec la question de l'ordre des constituants, certains aspects syntaxiques, s'observe également dans le jeu des formes verbales, plus précisément dans la possibilité de faire alterner le passé simple et le présent dans la conduite de la narration. Si cette alternance n'est pas une caractéristique spécifique aux textes anciens, elle est toutefois quasiment systématisée dans la plupart des textes narratifs en ancien français au point de devenir une routine discursive. Le travail de Rychner (1970) sur les séquences narratives dans La Mort Artu met bien en lumière les divers contextes dans lesquels s'opère le changement de forme verbale et les facteurs discursifs qui le justifient. Nous insisterons ici sur le point qui nous semble important pour le raisonnement que nous suivons. Les enchaînements qui mettent en jeu l'antéposition d'une proposition en quant ne semblent pas modifier de façon notable l'organisation temporelle qui caractérise les séquences de prédications non dépendantes en relation de parataxe. Dans la plupart des cas, le changement de temps, d'ordinaire l'introduction d'un présent de « narration » qui vient interrompre une série de passés simples, signale une nouvelle séquence 
discursive, un nouvel épisode de premier plan. Dans l'exemple suivant, la proposition au présent ( $s$ 'en passe outre) sert en quelque sorte de transition entre la rencontre et l'intervention de Brancillas auprès de l'empereur :

(25) à tant es vous que li premiers des sages vint. Il encontra le vallet que li serf enmenoient pendre. li uns ne dist mot à l'autre. Messires Bancillas s'en passe outre et vint au pié des degrez de la sale (Sept Sages, p. 10)

et voici que le premier des sages arriva. Il rencontra le jeune homme que les serviteurs emmenaient pour le pendre. L'un ne dit mot à l'autre. Monseigneur Bancillas ne s'arrête pas et vint au pied de l'escalier de la salle

Cette modification du temps de la narration peut également servir d'introduction à un passage de dialogue, comme dans :

(26) Li emperieres prist par la main destre son filz. puis monterent amont el palés. Li emperieres demande à son filz coment il li est. Li vallez li encline et riens ne li respont. Qu'est ce biax filz dit li emperieres [...] (p. 6)

L'empereur prit son fils par la main droite ; puis ils montèrent en haut du palais. L'impératrice demande à son fils ce qui lui arrive. Le jeune homme s'incline et ne réponds rien. Qu'est cela, beau fils, dit l'empereur

Il n'est guère étonnant que les subordonnées en quant jouent également ce rôle textuel de transition ; elles relèvent en effet généralement du second plan, dans la mesure où elles rappellent une partie du contenu du contexte antérieur, présentée comme le cadre de la deuxième séquence. Dans le premier des deux exemples suivants, le présent (giete) se trouve isolé, la séquence qu'il introduit étant construite sur un enchaînement de passés simples (descira, jeta), alors que, dans le deuxième extrait, le présent se maintient tout au long de l'unité textuelle (entort, se lieve, fet, espandent, tire) :

(27) Li vallez voloit garder l'enneur son père et la seue si ne dist mot. Quant l'empereriz vit qu'ele ne tireroit de lui mot si giete ses mains à uns dras de soie qu'ele avoit vestuz [...] si descira tout [...] si jeta ses mains à ses cheveus (Sept Sages, p. 7)

Le jeune homme voulait conserver l'honneur de son père et le sien; alors il ne dit mot. Quand l'impératrice vit qu'elle ne tirerait aucune parole de lui, alors elle prend dans ses mains les vêtements de soie [...] alors elle déchira [...] alors elle porta les mains à ses cheveux [...]

(28) Li sergent aporterent les premiers més [...] En ce que les vallez conmencierent à tranchier. la dame entort ses clés es frenges de la nape. puis se lieve et fet un grant pas avant, et les escuelles espandent desus la nape. Li sires fu moult iriez et la dame tire à soi ses clés [...] (p. 33)

Les serviteurs apportèrent les premiers plats [...] Pendant que les serviteurs commencèrent à couper les viandes, la dame entortille ses clés aux franges de la nappe, puis se lève et fait un grand pas en avant et les assiettes se renversent sur la nappe. Le seigneur fut très en colère et la dame reprend ses clés [...]

Dans ce type de progression, tout se passe comme si le passé simple de la subordonnée avait pour fonction de maintenir la continuité temporelle avec le contexte gauche, la rupture étant marquée par l'emploi du présent combiné avec celui d'une particule, comme en (27), ou de la ponctuation par le point, comme en (28). La subordonnée en quant, dans cette configuration discursive, ne se distingue guère des propositions non dépendantes qui remplissent le même rôle discursif souligné par le changement de forme verbale, changement qui est guidé par des raisons d'ordre discursif et non par des principes qui correspondraient à une supposée « concordance » des temps. Les paliers de traitement auxquels s'opère la gestion des temps de la narration évoluent en même temps que le système syntaxique et les propositions temporelles apparaissent, dans les textes que nous venons d'examiner, comme moins intégrées que le sont leurs équivalents en français moderne par exemple.

\section{Le rôle des propositions temporelles dans le déroulement de la narration}

Les deux propriétés que nous venons d'observer, l'ordre des constituants et le jeu des formes verbales, permettent de donner une place particulière aux subordonnées en quand, qui se 
distinguent ainsi à la fois des circonstancielles autres que les temporelles, mais également des subordonnées d'antériorité. C'est sur ce dernier point que les données de l'ancien français ne s'accordent pas avec la hiérarchisation proposée par Van Valin \& LaPolla. Lorsque l'on s'interroge sur les raisons de cette différence, l'observation du domaine discursif permet d'apporter des éléments de réponse.

Une explication pourrait en effet être cherchée dans le statut des propositions en quand dans la structure informationnelle qui caractérise le texte narratif en ancien français. Les contraintes syntaxiques liées à l'ordre V2 s'accompagnent de ce qui correspond à une simplification de l'échelle des degrés de dynamisme communicatif, la priorité étant donnée au marquage du thème propre, opposé à un ensemble rhématique peu différencié, sans que soient nettement signalés par des faits de position des éléments qui auraient par exemple la fonction de rhème ou de thème secondaire. Le schéma : subordonnée $+V(S) X$, qui caractérise les causales, les finales, les comparatives et les temporelles d'antériorité, fait ainsi entrer la proposition initiale dans une structuration informationnelle qui lui donne la fonction de thème ou, éventuellement, celle de rhème propre, ce deuxième cas correspondant à la valeur d'une construction clivée. Il n'en va pas de même pour les propositions en quand, dont nous avons constaté le statut périphérique ; placées en tête d'énoncé, elles n'entrent pas dans la hiérarchie de dynamisme communicatif organisée dans la prédication principale, mais, reprenant souvent des informations contenues dans le contexte gauche, elles jouent un rôle de constituant cadratif dont la portée s'exerce sur une séquence narrative plus ou moins longue. Dans l'extrait suivant, la subordonnée temporelle ouvre un récit qui s'étend sur huit propositions qui, à l'exception de la subordonnée en si que, s'enchaînent de façon paratactique, la fin de l'épisode étant signalé par l'emploi d'un nouveau cadratif(l'endemain) :

(29) Et quant il orent coureu par la terre et il s'en voudrent issir si troverent les destroiz moult forz. Et li blac dou pais se furent asamblé. et asamblerent a euls et leur firent moult grant domache. et de hommes et de chevax et furent moult pres de desconfire si que par vive force covint les chevaliers descendre a pié. Et par l'aide de dieu s'en revindrent toutes voies en l'ost. mais grant domage orent receu. L'endemain s'en parti le emperieres [...] (Villehardouin, p. 113)

Et quand ils eurent chevauché dans le pays et qu'ils voulurent en sortir, alors ils trouvèrent les détroits très bien défendus. Et les Blaques du pays se rassemblèrent et les attaquèrent et leur occasionnèrent de grandes pertes en hommes et en chevaux et ils furent près d'être vainqueurs si bien que les chevaliers furent forcés de mettre pied à terre. Et avec l'aide de Dieu ils revinrent rapidement au camp, mais ils avaient subi de grands dommages. Le lendemain l'empereur partit [...]

Cette valeur cadrative des temporelles n'est cependant pas le seul facteur à prendre en considération. Tout aussi important, sinon plus, que le fonctionnement large de leur portée, le rôle de ces prédications dans la progression de la narration ne doit pas être négligé. Ce qui semble l'emporter en effet, ce n'est pas tant l'information qu'elles apportent, du point de vue chronologique, sur la relation sémantique qui unit les propositions successives que la fonction discursive qu'elles remplissent. Alors que des formes comme einz que ou jusques à tant que mettent en saillance la valeur d'antériorité ou de postériorité, une forme comme quant fait passer au second plan la relation chronologique - du moins lorsque la subordonnée est placée en tête d'énoncé - pour mettre l'accent sur l'état de chose, qu'il s'agisse d'un processus ou d'un état résultant, qui constitue un élément de la séquence narrative. En ce sens, la succession quant $\mathrm{P}_{1} \mathrm{P}_{2}$ se révèle proche d'une situation de parataxe de type $\mathrm{P}_{1}$ et / si / lors $\mathrm{P}_{2}$ et un parallélisme nous semble pouvoir être établi entre les temporelles initiales et l'enchaînement paratactique des prédications. Dans les deux extraits suivants, où la succession temporelle s'accompagne d'une relation de causalité plus ou moins marquée, l'enchaînement des propositions est assuré par si, avec maintien du sujet ou introduction d'un sujet nouveau :

(30) Et cil de la cité qui moult l'avoient desiré issirent hors a procession si le virent moult volontiers (Villehardouin, p. 112) 
Et ceux de la cité qui l'avaient beaucoup attendu sortirent en processionet le virent avec beaucoup de plaisir

(31) Lors dona congié aus homes et aus fames qu'il ot rescous si s'en ala chascun la ou il vost (p. 103)

Alors il donna congé aux hommes et aux femmes qu'il avait secourus et chacun s'en alla où il voulut

Dans les exemples suivants, les mêmes relations sémantiques unissent les deux propositions, mais la première prend la forme d'une subordonnée temporelle, ce qui peut sans doute être justifié par le fait que le contenu a déjà été évoqué dans le contexte antérieur :

(32) Et quant il virent ce si en orent moult grant pitié. Et plorerent d'autre part moult tendrement.

Et quand ils virent cela, alors ils en eurent une grande pitié. Et ils pleurèrent très tendrement

(33) Quant il virent lor seigneurs et leur amis cheoir a leur piez. Il dirent qu'il em parleroient et se trerent a une part. (p. 26)

Quand ils virent leurs seigneurs et leurs amis tomber à leurs pieds, ils dirent qu'ils en parleraient et se retirèrent

Dans des passages de ce type, des «particules » comme si, lors ou et ne voient pas leur rôle limité à une corrélation avec quant ou avec d'autres subordonnants. Si c'était le cas, on pourrait considérer qu'à l'inverse de ce que nous essayons de montrer, les propositions placées en tête d'énoncé sont en fait étroitement rattachées au contexte de droite. Dans la mesure où les mêmes particules - dans les mêmes textes - fonctionnent comme des marqueurs de successivité, signalant la progression du premier plan, dans une configuration syntaxique de l'ordre de la parataxe, il nous semble que cette symétrie va dans le sens d'une faible intégration de certaines subordonnées qui apparaissent dans les mêmes contextes discursifs.

La conjonction quand, dans cet emploi discursif, possède ainsi une double valeur : vers l'aval du texte, elle signale la présence d'un constituant cadratif, alors que, vers l'amont, marquant à la fois la continuité et la rupture avec le contexte gauche, elle se rapproche d'un marqueur d'enchaînement comme et alors.

En nous attachant essentiellement aux subordonnées temporelles et à la question de l'ordre des constituants, nous avons voulu insister sur le fonctionnement particulier de certaines unités prédicatives placées en tête d'énoncé et montrer que les facteurs discursifs, en particulier le rôle de ces propositions dans le déroulement de la narration, dans le découpage en séquences textuelles, pouvait justifier, dans le continuum de subordination, leur faible degré d'intégration, proche de celui de la parataxe. Nous avons également observé, lié à ce fonctionnement, le changement de valeur de l'introducteur de la proposition, dont la fonction de «subordonnant» laisse la place à une valeur de marqueur discursif. Un élargissement de ces observations pourrait être opéré, nous semble-t-il, par le rapprochement du fonctionnement de ces prédications de celui des propositions topicalisées, introduites par des locutions comme quant à, qui se développent surtout durant la période du moyen français. Dans les deux cas, la position d'une unité prédicative en tête d'énoncé s'accompagne en effet d'une double rupture : rupture syntaxique avec le contexte de droite par la faible intégration dans une structure qui serait celle d'une «phrase complexe»; rupture discursive par l'introduction d'une nouvelle séquence narrative dans le cas des temporelles ou par le changement énonciatif, d'ordre polyphonique, qui caractérise les topicalisations. La prise en compte des indices qui sont privilégiés dans l'approche par continuum pourrait sans doute permettre, du moins pour l'ancienne langue, une unification de l'analyse de ces différentes structures prédicatives. D'un point de vue diachronique enfin, il convient de rappeler le rôle important que vont jouer la période du moyen français et, surtout, celle du français préclassique, pendant laquelle, d'une façon générale, disparaissent bon nombre de dissymétries qui caractérisaient l'ancien français. C'est en particulier la réorganisation de la zone préverbale et la disparition des contraintes concernant la position du sujet, changements 
dus à la disparition progressive du système V2, qui conduisent à une unification dans le fonctionnement des circonstancielles initiales, du moins pour certaines de leurs propriétés. On assiste ainsi à un double mouvement : alors que les circonstancielles les mieux intégrées voient leur fonctionnement se modeler sur celui des subordonnées périphériques, en particulier en ce qui concerne les faits de position, les propositions dotées d'un statut proche de la parataxe vont entrer progressivement dans le jeu des relations de dépendance de l'énoncé complexe.

\section{Références bibliographiques}

BERRENDONNER A. (2002), «Les deux syntaxes », Verbum 24, p. 23-35.

BURIDANT C. (2000), Grammaire nouvelle de l'ancien français, Paris, SEDES.

Combettes B. (2002), « Aspects de la grammaticalisation de la phrase complexe en ancien et en moyen français », Verbum 24, 109-128.

CoRminboeuf G. (2009), L'expression de l'hypothèse en français contemporain, entre hypotaxe et parataxe, Bruxelles : De Boeck-Duculot.

DebaisieuX J.-M. (2013), «Quelques problèmes posés par la notion de subordination », in J.-M. Debaisieux (éd.), Analyses linguistiques sur corpus : subordination et insubordination en français, Traité IC2, Hermès-Lavoisier.

DeBAISIEUX, J.-M., (dir.), Analyses linguistiques sur corpus. Subordination et insubordination en français, Paris, Editions Lavoisier, 37-59.

HAIMAN J. (1978), "Conditionals are topics", Language 54, 564-589.

HAIMAn J. \& ThOMPSON S. A. (eds.) (1988), Clause Combining in Grammar and Discourse, Amsterdam: John Benjamins.

HARRIS M. (1988), "Concessive clauses in English and Romance", in J. Haiman \& S. A. Thompson (eds), Clause Combining in Grammar and Discourse, Amsterdam: John Benjamins, p. 71-99.

KÖNIG E. \& VAN DER AUWERA J. (1988), "Clause integration in German and Dutch conditionals, concessive conditionals, and concessive", in J. Haiman \& S. A. Thompson (eds.) (1988), Clause Combining in Grammar and Discourse, Amsterdam: John Benjamins, 101-133.

LEHMANN C. (1988), "Towards a typology of clause linkage", in J. Haiman \& S. Thompson (eds.) (1988), Clause Combining in Grammar and Discourse, Amsterdam: John Benjamins, 181-225

MARChEllo-Nizia C. (1995), L'évolution du français. Ordre des mots, démonstratifs, accent tonique, Paris : Armand Colin.

MATthiesSEN C. (2002), "Combining clauses into clause complexes", in J. Bybee \& M. Noonan (eds), Complex Sentences in Grammar and discourse, Amsterdam : John Benjamins, 237-322.

Matthiessen C. \& ThOMPSON S. A. (1988), "The structure of discourse and "subordination", in J. Haiman \& S. A. Thompson, (eds), (1988), Clause Combining in Grammar and Discourse, Amsterdam: John Benjamins, 275-329.

PIERRARD M. (1993), «Subordination, dépendance et hiérarchie », Travaux de Linguistique 27, 13-28.

PREVost S. (2001), La postposition du sujet en Français aux XVe et XVIe siècles, Paris : CNRS Éditions.

RYCHNER J. (1970), L'articulation des phrases narratives dans la Mort Artu, Genève : Droz.

SCHIFFRIN D. (1992), "Conditionals as topics", Linguistics 30, 165-197.

SKÅRUP P. (1975), Les premières zones de la proposition en ancien Français. Essai de syntaxe de position, København : Akademisk.

TORTERAT F. (2000), «Et en emploi "syndético-hypotactique" : hypothèse sur une jonction implicite en ancien et en moyen français », BSL 95, 183-202.

VAn VAlin R. \& Lapolla R. (1997), Syntax. Structure, meaning and function, Cambridge: Cambridge University Press.

VerSTRAETE J.-C. (2007), Rethinking the coordinate subordinate dichotomy, Berlin, Mouton de Gruyter. 